\title{
Management of Abdominal Ewing's Sarcoma: A Single Institute Experience
}

\author{
Ambarish Chatterjee $^{1} \cdot$ Shraddha Patkar ${ }^{1}$ (1) $\cdot$ Nilendu Purandare $^{2} \cdot$ Smruti Mokal $^{3} \cdot$ Mahesh Goel $^{1}$
}

Received: 18 April 2021 / Accepted: 27 July 2021/Published online: 3 August 2021

(C) Indian Association of Surgical Oncology 2021

\begin{abstract}
Ewing's sarcoma (ES)/primitive neuroectodermal tumors (PNETs) are a rare group of tumors commonly arising from bones, uncommonly from soft tissues, and rarely from abdomen. The aim of the study was to analyze the outcome (recurrence-free survival[RFS]), patient characteristics, role of FDG-PET (fluorodeoxyglucose positron emission tomography) computerized scan, chemotherapy and radiation, and prognostic factors. We retrospectively studied patients diagnosed with abdominal ES/ PNET and treated surgically between June 2005 and November 2019. Ten patients were included in the study, with a median age of 36.5 years (19-46 years). The median follow-up was 25 months (3-178 months). The site of origin was the retroperitoneum, small bowel, and abdominal wall in six, two, and two patients, respectively. $70 \%$ of patients were treated with induction chemotherapy. R0 resection was achieved in $90 \%$ of patients. With chemotherapy, there was significant reduction in tumor size $(\mathrm{p}=0.034)$ with non-significant reduction in SUV $\max (\mathrm{p}=0.31)$. The 1- and 2-year RFS were $88.90 \%$ and $76.20 \%$, respectively. Pathological peritoneal metastasis and ability to achieve R0 resection were prognostic factors affecting RFS. These patients must be offered multimodality treatment. Induction chemotherapy significantly reduces the tumor size. Pathological peritoneal metastasis and ability to achieving R0 resection significantly affect survival.
\end{abstract}

Keywords Ewing's sarcoma $\cdot$ R0 resection $\cdot$ Induction chemotherapy $\cdot$ FDG-PET scan

\section{Background}

Ewing's sarcoma (ES)/primitive neuroectodermal tumors (PNETs) are a well-known mesenchymal tumor belonging to a group of small round cell tumors (SRCTs) with simple sarcoma-specific genetic alterations between genes of TET/FET family and Erythroblast Transformation Specific (ETS) family with translocation of EWSR1 gene on chromosome $22 q 12 t(11 ; 22)(q 24 ; q 12)$ occurring in $90 \%$ of cases [1]. ES/PNET expresses CD99 on its membrane that differentiates it from other SRCTs [2]. ES is commonly seen as a primary bone tumor, although it often arises from soft tissues (extra-osseous ES [EES]) [3]. ES/PNETs belong to

Shraddha Patkar

drshraddhapatkar@gmail.com

1 Gastrointestinal and Hepatopancreaticobiliary Services, Department of Surgical Oncology, Tata Memorial Hospital, Homi Bhabha National Institute, Mumbai 400012, India

2 Department of Nuclear Medicine, Tata Memorial Hospital, Homi Bhabha National Institute, Parel, Mumbai 400012, India the same spectrum of neoplasms known as Ewing sarcoma family of tumors (EFT) which also includes malignant small cell tumor of the chest wall (Askin tumor) and atypical ESS [4-6]. ES represents the least differentiated and PNET represents the most differentiated tumors [7]. Abdominal ES/ PNETs are rare with few case reports in literature [8-20]. Abdominal ES/PNETs are often confused with other SRCTs such as embryonal rhabdomyosarcomas, neuroblastomas, and lymphomas. This makes it even more important for accurate diagnosis to provide evidence-based multimodality management for optimal outcomes.

The primary objective was to study the management of abdominal ES along with a review of literature.

3 Department of Biostatistics, Clinical Research Secretariat, Tata Memorial Hospital, Homi Bhabha National Institute, Parel, Mumbai 400012, India 


\section{Methods}

\section{Data Source and Patient Population}

The demographic, clinical, radiological, treatment, histopathological, and survival data of patients were obtained retrospectively from hospital's electronic medical records. The inclusion criteria were:

1. Biopsy proven abdominal ES/PNET disease

2. Age $\geq 14$ years

3. Eastern Cooperative Oncology Group (ECOG) performance status $0-1$

4. Surgical treatment in the institute

The exclusion criteria were:

1. Poor performance status $(\mathrm{ECOG} \geq 2)$

2. Presence of metastatic disease

Ten patients identified and registered between June 2005 and November 2019 were included in the study. The diagnosis was established by histopathological examination, and immunohistochemical analysis. Molecular study was not routinely performed for diagnosis in the institution. Disease was staged using 18-Fluorodeoxyglucose Positron Emission Tomography with Contrast Enhanced Computed Tomography (FDG-PETCECT) scan (except for two patients for whom CECT scan was done) and bone marrow biopsy was obtained in patients treated till 2010 (four patients). As a part of Institutional policy, PETCECT scan was selectively used from 2005 to 2010. After 2010, PETCECT scan was incorporated as a staging tool and subsequently bone marrow biopsies were omitted. Clinical tumor (cT) size (defined by its largest dimension) and maximum standardized uptake value (SUV max) were recorded and compared before and after chemotherapy. All patients were discussed in multidisciplinary joint clinics (MDJCs) involving surgical oncologist, medical oncologist, radiation oncologist, pathologist, nuclear medicine consultant, and radiologist. The chemotherapy regimen followed was EFT 2001 protocol [21, 22]. Post-chemotherapy, surgery was performed after clinical and radiological assessment through MDJC. Post-surgical resection, patients were continued on maintenance chemotherapy as per protocol. Complications were graded and recorded as per Clavien-Dindo classification of surgical complications [23]. Adjuvant radiation was given to patients based on histopathological findings, tumor volume, margin status, and response to chemotherapy. All patients were followedup at three-monthly intervals for the first three years, sixmonthly intervals for the next two years, and annually thereafter. In view of COVID-19 pandemic, all follow-ups after February 2020 were done via telephonic calls.

\section{Statistical Analysis}

The date of registration at the hospital was considered as date of diagnosis for statistical purposes. RFS was defined as interval between dates of diagnosis and appearance of first recurrence. RFS was calculated using the Kaplan-Meier method and compared using the log rank test. A univariate Cox regression hazard model analysis was used to analyze any prognostic factors of outcome and the hazard ratio (HR), relevant $95 \%$ confidence intervals (CIs) were calculated. $\mathrm{P}<0.05$ was considered to indicate a statistically significant difference. Wilcoxon signed rank test was used to test whether there was any significant change between pre-chemotherapy FDG-PETCECT scan (cT) size and SUV max due to chemotherapy. Spearman rho coefficient test was used to derive correlation between pathological tumor size (pT) with post-chemotherapy cT size and post-chemotherapy SUV max. The database of all patients in the review was compiled and analyzed using SPSS v.21 software (IBM Corp.).

\section{Results}

\section{Baseline Patient Characteristics and Staging}

Ten patients were analyzed in this study and the baseline patient characteristics, staging investigations, baseline cT size, and SUV max and post-chemotherapy cT size and SUV max are shown in Table 1. There were six males and four females with a median age of diagnosis of 36.5 years (range 19 to 46 years). The site of origin was retroperitoneum (six patients), small bowel (two patients), and abdominal wall (two patients).

\section{Surgical Aspects}

All patients (except one - patient E) underwent R0 resection of which five patients required multivisceral resections [Table 2 (A)]. The median blood loss was $450 \mathrm{ml}$ (range: 100-1900 ml) and only three patients (A, D, and E) had Clavien-Dindo grade-1 complications. Table 2 (A) shows the histological features of these patients. The median pathological tumor size (pT) was $13.50 \mathrm{~cm}$ (range $2.20 \mathrm{~cm}$ to $20 \mathrm{~cm}$ ). Five patients had disease involving the resected organs (B, E, F, G, and I). Two patients (E and I) had peritoneal metastasis, patient I underwent $\mathrm{R} 0$ resection while patient E underwent R2 resection (95\% tumor was grossly debulked, residual multiple small deposits were present on parietal peritoneum and sigmoid colon mesentery). 


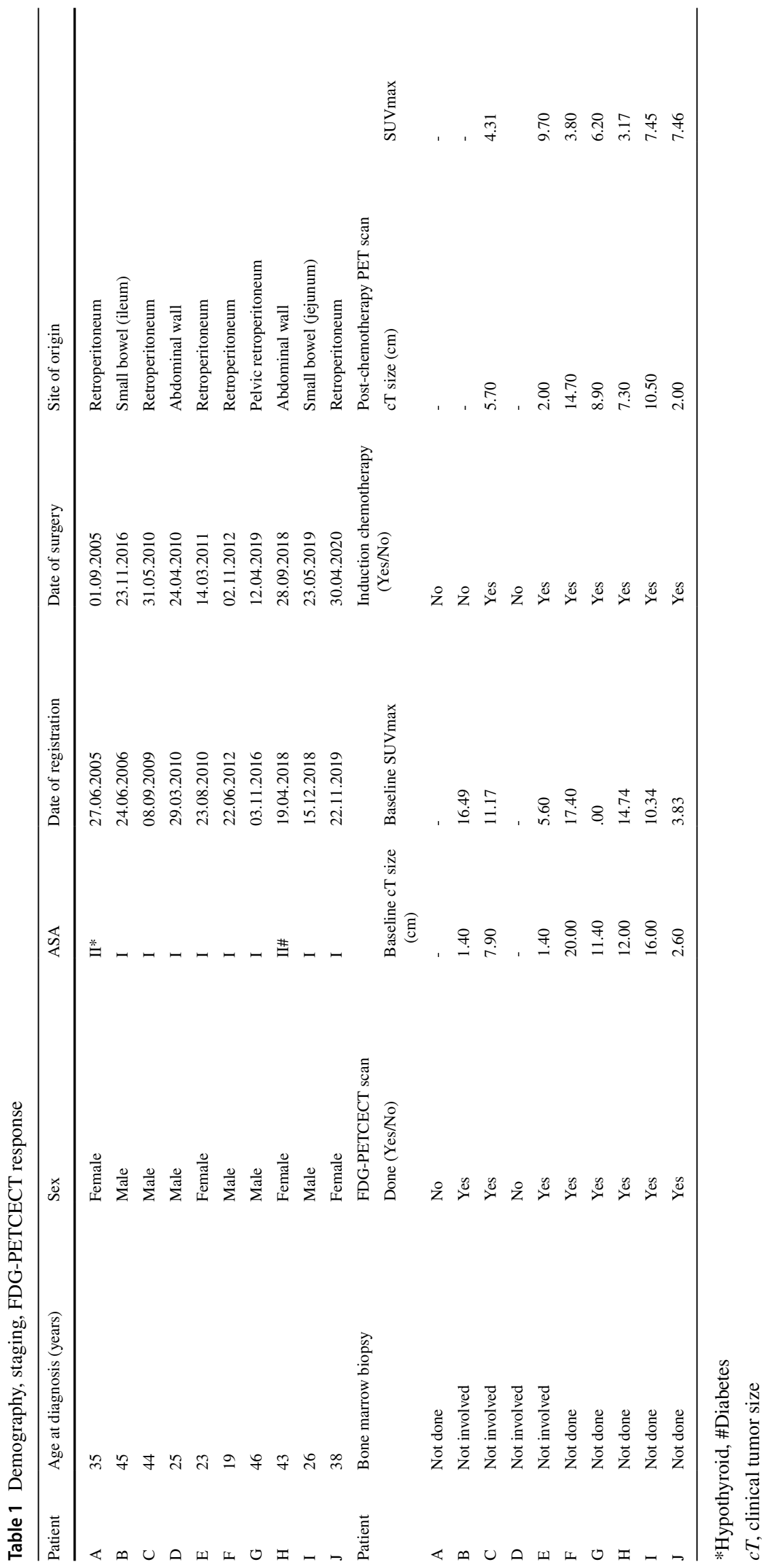




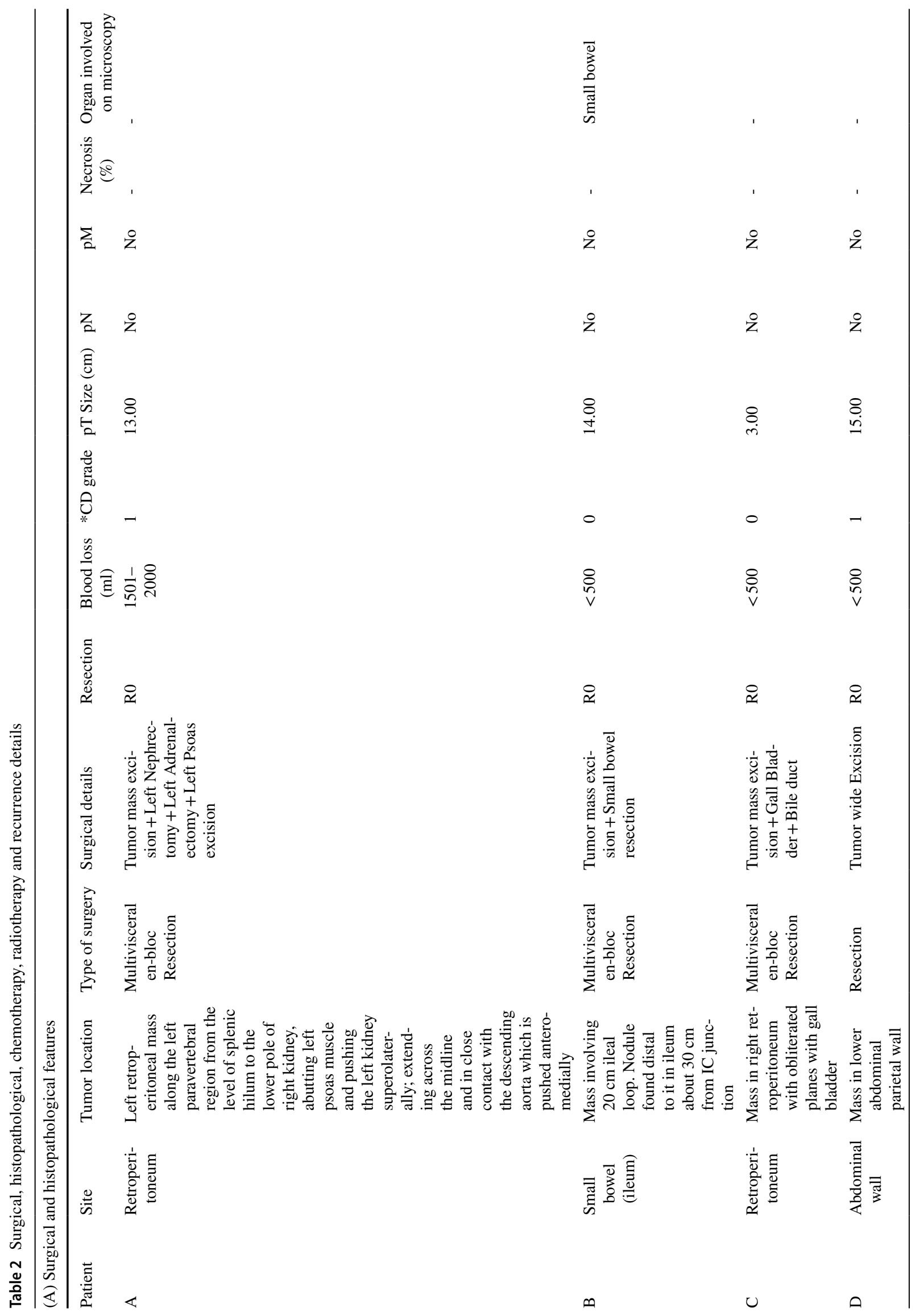




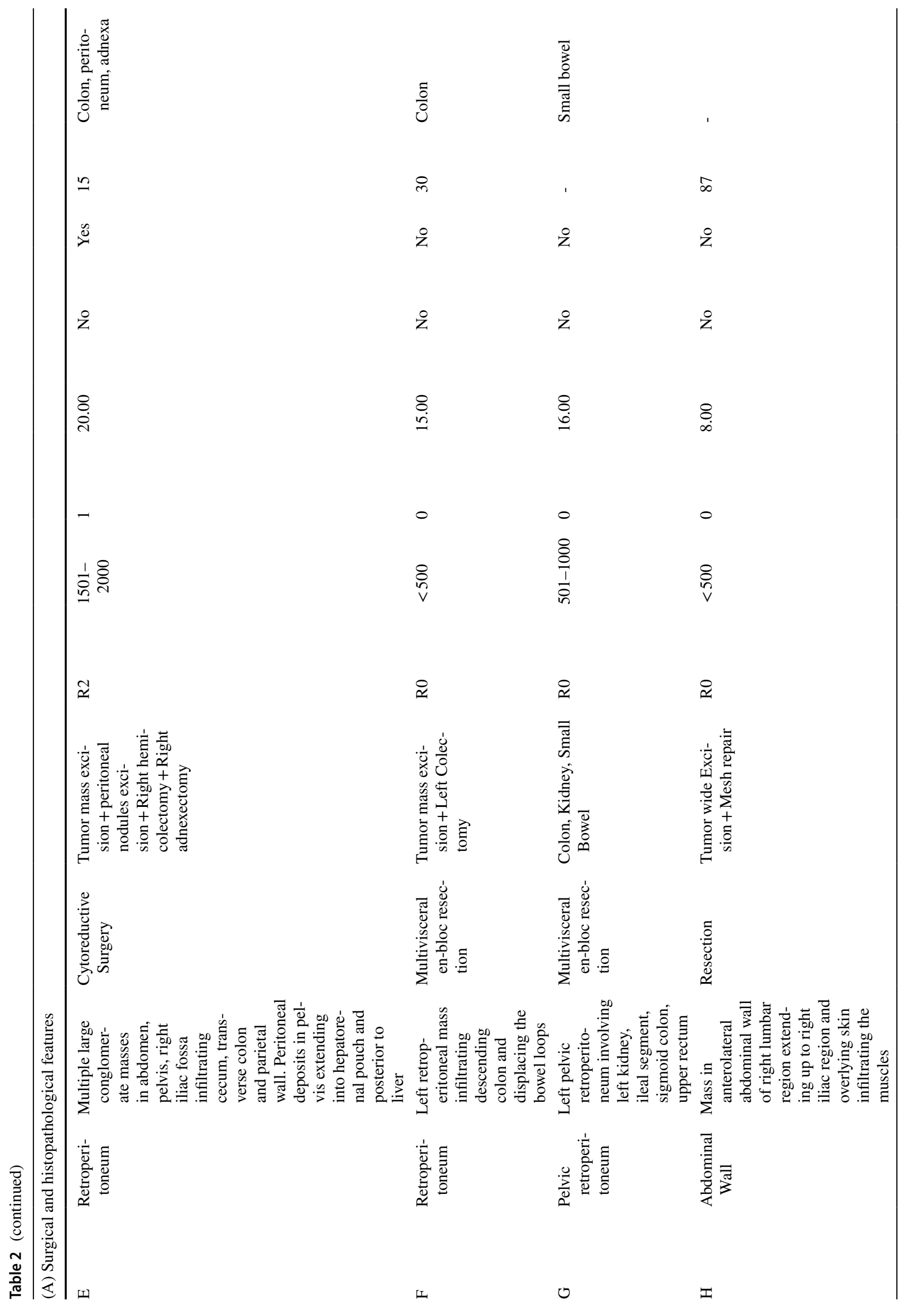




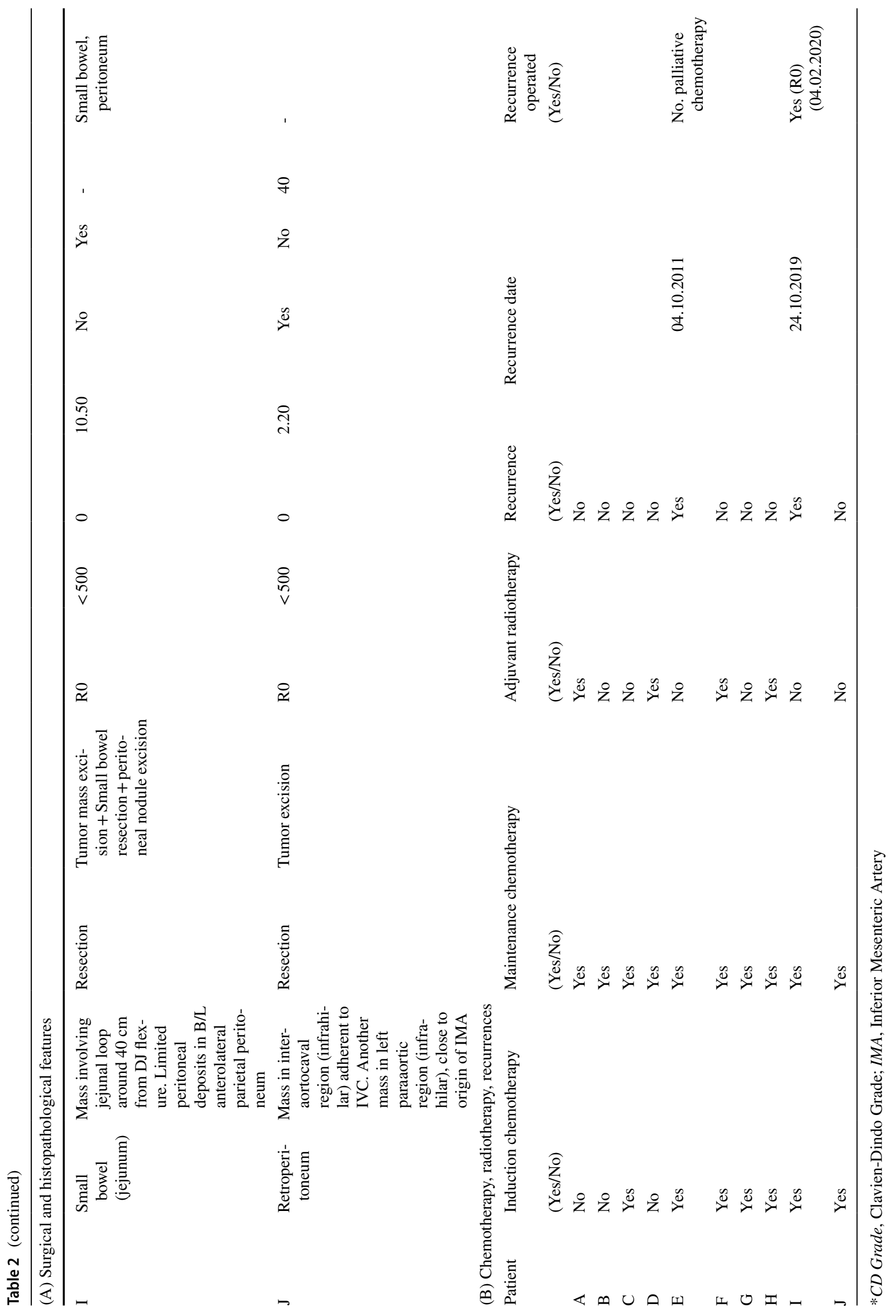


Table 2 (B) provides the details of induction, maintenance chemotherapy, and adjuvant radiotherapy received by these patients. Patient B underwent emergency surgery in view of intra-luminal bleeding. The patient presented with anemia (hemoglobin $5.1 \mathrm{gm} / \mathrm{dL}$ ) and $12 \times 10 \mathrm{~cm}$ mass. Two patients (E and I) had recurrences [Table 2 (B)], patient I was rechallenged with chemotherapy and underwent cytoreduction to achieve R0 resection whereas patient $\mathrm{E}$ was deemed unresectable and continued palliative chemotherapy.

\section{FDG-PETCECT Scan}

Eight patients underwent baseline FDG-PETCECT scan, and post-chemotherapy FDG-PETCECT scan of one patient was not available, hence, seven patients' data were available for analysis (Table 1). Chemotherapy did not cause any statistical significant difference between pre-chemotherapy and post-chemotherapy median SUV max $(\mathrm{p}=0.31)$; however, there was significant reduction in $\mathrm{cT}$ size with chemotherapy $(p=0.034)$. On univariate analysis, post-chemotherapy SUV max and cT size were not prognostic or predictive of recurrence. There was a weak positive correlation between $\mathrm{pT}$ size and cT size $(r h o=0.252 ; \mathrm{p}=0.585)$ and between $\mathrm{pT}$ size and post-chemotherapy SUV $\max (r h o=0.500 ; p=0.253)$.

\section{Outcomes and Prognostic Factors}

The median follow-up was 25 months (range: 3-178 months). The 1- and 2-year RFS of the cohort was $88.90 \%$ (95\% CI: 70.60 to $100 \%$ ) and $76.20 \%$ (95\% CI: 52.1 to $100 \%$ ), respectively (Fig. 1). Overall survival (OS) could not be calculated, as there were no events in the series. The only prognostic factors associated with recurrence were presence of pathological peritoneal disease/metastasis $(\mathrm{p}=0.0026)$ and ability to achieve $\mathrm{R} 0$ resection $(\mathrm{p}=0.11)$ (Table 3). Two patients developed recurrences, patient $\mathrm{E}$ had recurrences as retroperitoneal and mesenteric disease with subcutaneous umbilical nodule, and patient I developed recurrence in the form of peritoneal deposits in diaphragmatic peritoneum, pelvis, and transverse mesocolon.

\section{Discussion}

There are subtle biological differences between ES/PNET arising from bones and EES that lead to varying presentation in terms of age, tumor characteristics, clinical features, treatment strategies and outcomes [24-26]. ES/PNETs most often present as primary skeletal tumor in the trunk or axial skeleton in adolescents and young adults. Due to the rarity of EES the available literature is scarce and limited to few case reports. However, in recent years, there has been an increase in case reports which might be because of the recognition of EES and abdominal ES/PNET as a separate entity and improved cytogenetic and molecular diagnostic methods. Hence, it becomes necessary to identify this rare group of patients early in the course of disease.

Patient characteristics in EES differ than in those with skeletal ES/PNET. In our study of 10 patients (six males, four females), the median age at diagnosis was 36.5 years. The median age across different case reports of abdominal ES/PNET have been in similar age-groups ( $15-35$ years) $[8$, 10, 12-15, 20, 21]. National Cancer Database (NCDB) and Surveillance, Epidemiology, and End Results (SEER) database studies show that the median age of patients at diagnosis of EES is older than its skeletal counterparts and has female predilection. EES patients are more likely to be $<5$ or $\geq 35$ years at diagnosis $[25,26]$. The average tumor size did not differ between skeletal ES/PNET and EES [25].

ES/PNET tumors have increased rate of glycolysis and hence the cells retain avidity. Meta-analysis of 23 studies by Huang et al. showed sensitivity and specificity that were $86 \%$ and $80 \%$, respectively with higher sensitivity and specificity (93\% and 90\% respectively) for detecting recurrences [27]. Hawkins et al. [28] and Salem et al. [29] conducted retrospective studies and showed that FDG-PETCECT scan correlates with histologic response, is prognostic and predictive of survival outcomes. In our study, there was significant difference between pre-chemotherapy and post-chemotherapy cT size (median cT size $11.40 \mathrm{~cm}$ vs $7.30 \mathrm{~cm}$ respectively, $\mathrm{p}=0.034$ ). However, the SUV max did not statistically differ in pre-chemotherapy and post-chemotherapy setting (10.34 vs 4.60 respectively, $p=0.31$ ). This could either be because of less number of patients, or biological differences in behaviors between abdominal ES/PNETs with its skeletal counterparts. We also did not find a strong positive correlation between $\mathrm{pT}$ size and cT size or post-chemotherapy SUV max. However, chemotherapy did bring about significant reduction in $\mathrm{cT}$ size $(\mathrm{p}=0.034)$.

The NCDB retrospective cohort study analyzed $2660 \mathrm{ES} /$ PNET patients (2004-2014) containing 1682 (63.2\%) skeletal ES/PNET and 978 (36.8\%) ESS patients. They concluded that EES patients have well-differentiated tumors, more likely to be in stage I disease with no significant difference in metastatic disease at diagnosis, perioperative outcome and no significant difference in OS of the entire cohort (median OS: 47.5 months for skeletal ES/PNET and 48.2 months for EES) [25]. In the SEER database of 2202 patients, the OS for localized ESS was superior to skeletal ES/PNET in both 5- and 10 -year OS; $69.7 \%$ vs $62.6 \%$ and $65.2 \%$ vs $55.3 \%$, respectively. However, when localized and metastatic ES/ PNETs were analyzed together or in metastatic disease alone, the OS is similar in ESS and skeletal ES/PNET. They also suggested that patients with ESS had worse OS in the first 24 months but better OS after 24 months as compared 
Fig. 1 Recurrence-free survival

Recurrence Free survival
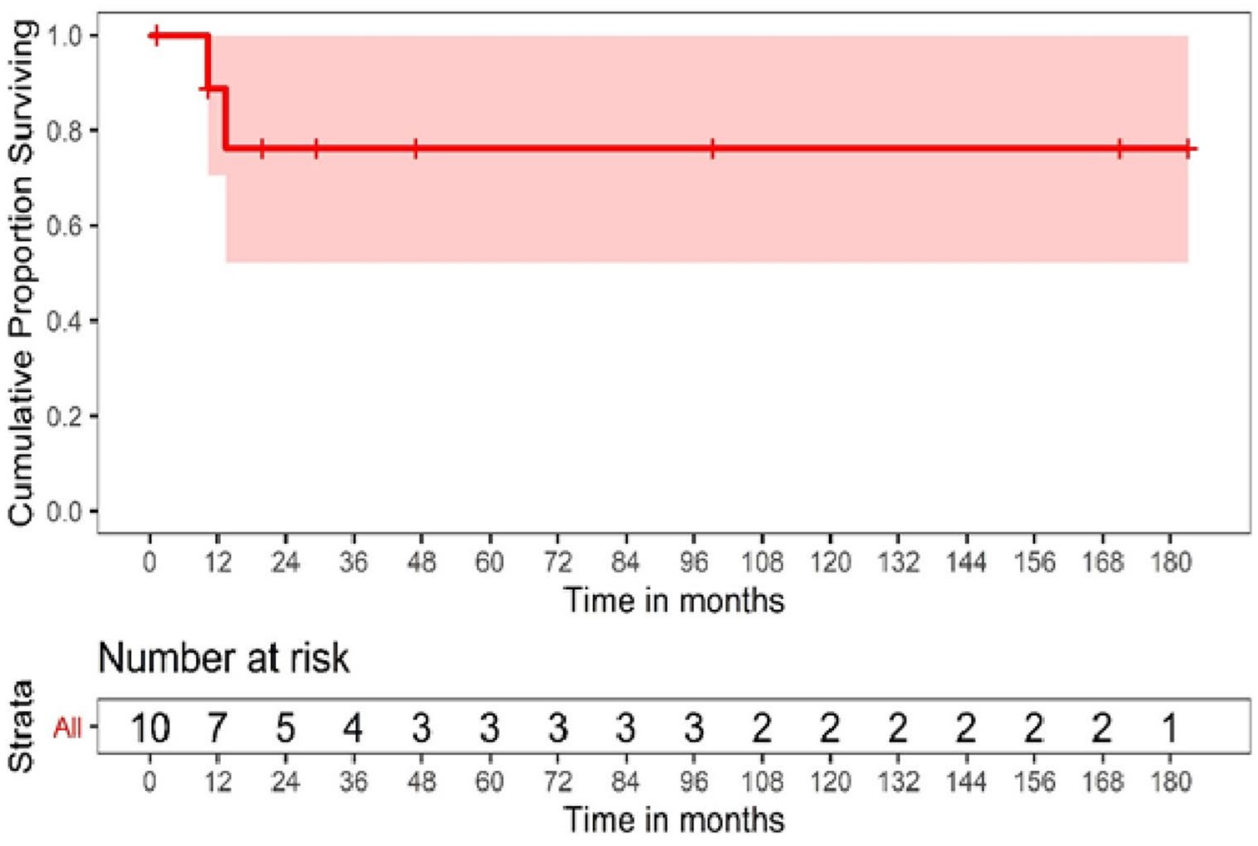

to skeletal ES/PNET patients, after controlling for other known prognostic factors suggesting that extra-skeletal site has greater impact on the long-term outcomes [26]. Studies with small number of patients also showed no significant differences in survival outcomes $[30,31]$.

In our study, the 1- and 2-year RFS were $88.90 \%$ and $76.20 \%$, respectively. This survival figures are impressive since they represent patients who were operated upon and did not include metastatic/inoperable patients. With multimodality uniform treatment comprising of induction chemotherapy, surgery, maintenance chemotherapy, and

Table 3 Prognostic factors

\begin{tabular}{lll}
\hline & Prognostic factors & P value \\
\hline 1 & Sex & 0.86 \\
3 & Subsite of origin & 0.65 \\
4 & Multivisceral resection & 0.13 \\
5 & Nephrectomy & 0.41 \\
6 & Colectomy & 0.73 \\
7 & Small bowel resection & 0.56 \\
8 & Peritoneal disease & $\mathbf{0 . 0 0 2 6}$ \\
10 & R0 vs R2 resection & 0.11 \\
11 & Induction chemotherapy & 0.56 \\
12 & Adjuvant radiation & 0.24 \\
15 & Pathological metastasis & $\mathbf{0 . 0 0 2 6}$ \\
16 & Post-chemotherapy PET cT size & 0.558 \\
17 & Post-chemotherapy PET SUVmax & 0.149 \\
18 & Pathological tumor size (pT) & 0.46 \\
\hline
\end{tabular}

Values in bold are statistically significant adjuvant radiation (when indicated) superior outcomes can be expected.

ES/PNET is considered a systemic disease with majority patients have subclinical metastatic disease at the time of diagnosis even in the absence of overt metastasis. Modern treatment protocols utilize initial chemotherapy (induction/neoadjuvant) followed by local treatment and further chemotherapy (maintenance/adjuvant). Induction chemotherapy is important to downstage the tumor to achieve $\mathrm{R} 0$ resection, improve local control and control metastatic disease [32-34]. EES patients respond to the same chemotherapy regimens as skeletal ES and should be treated similarly [35-38]. The chemotherapy protocols have evolved over years with the IESS-I-III studies leading to the current regimen which constitutes alternating cycles of ifosfamide and etoposide (I/E) to vincristine, adriamycin, and cyclophosphamide (VAC) backbone (VAC/IE) [39-41].

All of our patients received chemotherapy as per EFT 2001 protocol. Three patients did not receive induction chemotherapy (patient B underwent emergency surgery in view of bleeding while the reason for patients A and D were not available).

ES/PNET is a radiosensitive tumor. Radiation is more commonly used in skeletal ES/PNET as compared to EES [26]. There are no randomized control trials comparing surgery and radiation. Surgery is the preferred modality of choice for local control if the tumor can be resected with negative margins without excess morbidity and with a reasonable functional result. Surgery also avoids the risk of secondary radiation-induced sarcomas and provides for analysis of degree of necrosis. However, if a tumor is unresectable following induction chemotherapy, the patient should be referred for definitive radiation. Data from skeletal ES/ 
PNET tumors provide for the indications of adjuvant radiation in the following settings:

i. Bulky tumors in difficult sites

ii. Macro- or microscopic residual viable tumor after surgery

iii. Positive or inadequate surgical margins

iv. High risk chest wall tumors

In our series, $4 / 10$ patients (40\%) received adjuvant radiation, two patients had retroperitoneal origin, and two had abdominal wall origin.

In our study, presence of peritoneal metastasis was found to be statistically significant factor $(p=0.0026)$ and $R 0$ resection $(\mathrm{p}=0.11)$ was found to be non-significant factor but with trend towards significance (Table 3 ).

The limitations of our study are its small number of cases, the lack of events observed, and lack of long-term data. However, as we have limited our study to exclusively include abdominal ES/PNETs who underwent surgical resection, this number is still significant. The lack of events observed in our study shows that this subset of patients have good prognosis with multimodality approach, aggressive surgical resection, and salvage surgery whenever feasible.

\section{Conclusion}

Abdominal ES/PNET being a rare entity requires special attention with accurate histopathological diagnosis, IHCs and molecular studies (in special cases) and baseline imaging with FDG-PETCECT. Tumor size on FDG-PETCECT is predictive of tumor response to chemotherapy. All patients must be offered multimodality treatment including induction chemotherapy, surgery, and maintenance chemotherapy and adjuvant radiation when indicated. Every attempt to obtain R0 resection must be made and induction chemotherapy significantly reduces the tumor size. Presence of pathological peritoneal metastasis and inability to achieve $\mathrm{R} 0$ resection affect survival significantly.

Author Contribution 1) Substantial contributions to the conception or design of the work; or the acquisition, analysis, or interpretation of data for the work or the creation of new software used in the work:

Ambarish Chatterjee, Shraddha Patkar, Nilendu Purandare, Smruti Mokal

2) Drafting the work or revising it critically for important intellectual content: Goel

Ambarish Chatterjee, Shraddha Patkar, Nilendu Purandare, Mahesh

3) Approved the version to be published:

Shraddha Patkar, Nilendu Purandare, Mahesh Goel
4) Agreement to be accountable for all aspects of the work in ensuring that questions related to the accuracy or integrity of any part of the work are appropriately investigated and resolved:

Shraddha Patkar

Data and Mmaterials Availability Available with the corresponding author on request.

Code Availability NA

\section{Declarations}

Ethics Approval and Consent to Participate Ethical approval was waived by the local Ethics Committee of the Institute in view of the retrospective nature of the study and all the procedures being performed were part of the routine care. Verbal informed consent was obtained to data acquisition.

Consent for Publication The participant has consented to the submission of the article to the journal.

Conflict of Interest The authors declare no competing interests.

\section{References}

1. Wei S, Siegal GP (2014) Round cell tumors of bone: an update on recent molecular genetic advances. Adv Anat Pathol 21(5):359-372

2. Bahrami A, Truong LD, Ro JY (2008) True identity by immunohistochemistry. Arch Pathol Lab Med 132:23

3. Angervall L, Enzinger FM (1975) Extraskeletal neoplasm resembling Ewing's sarcoma. Cancer 36(1):240-251

4. Askin FB, Rosai J, Sibley RK et al (1979) Malignant small cell tumor of the thoracopulmonary region in childhood: a distinctive clinicopathologic entity of uncertain histogenesis. Cancer 43(6):2438-2451

5. Llombart-Bosch A, Terrier-Lacombe MJ, Peydro-Olaya A, Contesso G (1989) Peripheral neuroectodermal sarcoma of soft tissue (peripheral neuroepithelioma): a pathologic study of ten cases with differential diagnosis regarding other small, round-cell sarcomas. Hum Pathol 20(3):273-280

6. Grier HE (1997) The Ewing family of tumors. Ewing's sarcoma and primitive neuroectodermal tumors. Pediatric Clinics of North America 44(4):991-1004

7. Diagnostic Histopathology of Tumors: 2 Volume Set - 4th Edition [Internet]. [cited 2020 Nov 10]. Available from: https://www.elsev ier.com/books/diagnostic-histopathology-of-tumors-2-volume-set/ fletcher/978-1-4377-1534-7

8. Jimenez RE, Folpe AL, Lapham RL et al (2002) Primary Ewing's sarcoma/primitive neuroectodermal tumor of the kidney: a clinicopathologic and immunohistochemical analysis of 11 cases. Am J Surg Pathol 26(3):320-327

9. Johnson AD, Pambuccian SE, Andrade RS et al (2010) Ewing sarcoma and primitive neuroectodermal tumor of the esophagus: report of a case and review of literature. Int J Surg Pathol 18(5):388-393

10. E C, Ee G, Hc H et al. (2011) Primitive neuroectodermal tumor of the liver: a case report. Case Rep Med 2011:748194-748194

11. Ren Y-L, Tang X-Y, Li T (2011) Ewing sarcoma-primitive neuroectodermal tumor of the uterus: a clinicopathologic, 
immunohistochemical and ultrastructural study of one case. Arch Gynecol Obstet 283(5):1139-1143

12. Li T, Zhang F, Cao Y et al (2017) Primary Ewing's sarcoma/ primitive neuroectodermal tumor of the ileum: case report of a 16-year-old Chinese female and literature review. Diagn Pathol 12(1):37

13. Bose P, Murugan P, Gillies E, Holter JL (2012) Extraosseous Ewing's sarcoma of the pancreas. Int J Clin Oncol 17(4):399-406

14. Abi-Raad R, Manetti GJ, Colberg JW et al (2013) Ewing sarcoma/ primitive neuroectodermal tumor arising in the adrenal gland. Pathol Int 63(5):283-286

15. Adair A, Harris SA, Coppen MJ, Hurley PR (2001) Extraskeletal Ewings sarcoma of the small bowel: case report and literature review. J R Coll Surg Edinb 46(6):372-374

16. Shek TW, Chan GC, Khong PL et al (2001) Ewing sarcoma of the small intestine. J Pediatr Hematol Oncol 23(8):530-532

17. C B, Gp S, K P et al. (2006) Primitive neurectodermal tumors: a case of extraosseous Ewing's sarcoma of the small intestine and review of the literature. J BUON 11(4):519-22

18. Prasertvit S, Stoikes N (2013) A rare case of ewing's sarcoma of the small intestine. Am Surg 79(2):E78-79

19. Vignali M, Zacchè MM, Messori $\mathrm{P}$ et al (2012) Ewing's sarcoma of the small intestine misdiagnosed as a voluminous pedunculated uterine leiomyoma. Eur J Obstet Gynecol Reprod Biol 162(2):234-235

20. Askri A (2008) CASE REPORT: Extraskeletal Ewing sarcoma of the abdominal wall. Cancer Imaging 8(1):156-158

21. Thyavihally YB, Tongaonkar HB, Gupta S et al (2008) Primitive neuroectodermal tumor of the kidney: a single institute series of 16 patients. Urology 71(2):292-296

22. Parambil BC, Vora T, Sankaran H et al. (2020) Outcomes with nondose-dense chemotherapy for Ewing sarcoma: a practical approach for the developing world. Pediatr Blood Cancer [Internet] [cited 2021 Jan 5];67(11). Available from: https://onlinelibr ary.wiley.com/doi.org/10.1002/pbc.28604

23. Dindo D, Demartines N, Clavien P-A (2004) Classification of surgical complications: a new proposal with evaluation in a cohort of 6336 patients and results of a survey. Ann Surg 240(2):205-213

24. Bovée JVMG, Hogendoorn PCW (2010) Molecular pathology of sarcomas: concepts and clinical implications. Virchows Arch 456(2):193-199

25. Lynch AD. Extraskeletal versus skeletal Ewing sarcoma in the adult population: controversies in care. Surgical Oncology. 2018;7.

26. Applebaum MA, Worch J, Matthay KK et al (2011) Clinical features and outcomes in patients with extraskeletal ewing sarcoma. Cancer 117(13):3027-3032

27. Huang T, Li F, Yan Z et al. (2018) Effectiveness of 18F-FDG PET/ $\mathrm{CT}$ in the diagnosis, staging and recurrence monitoring of Ewing sarcoma family of tumors: a meta-analysis of 23 studies. Medicine 97(48):e13457

28. Hawkins DS, Schuetze SM, Butrynski JE et al (2005) $\left[{ }^{18}\right.$ F] Fluorodeoxyglucose positron emission tomography predicts outcome for Ewing sarcoma family of tumors. JCO 23(34):8828-8834
29. Salem U, Amini B, Chuang HH et al (2017) ${ }^{18}$ F-FDG PET/CT as an indicator of survival in Ewing sarcoma of bone. J Cancer 8(15):2892-2898

30. Pradhan A, Grimer RJ, Spooner D et al (2011) Oncological outcomes of patients with Ewing's sarcoma: is there a difference between skeletal and extra-skeletal Ewing's sarcoma? J Bone Joint Surg Br 93(4):531-536

31. Orr WS, Shannon Orr W, Denbo JW et al. Analysis of prognostic factors in extraosseous Ewing sarcoma family of tumors: review of St. Jude Children's Research Hospital experience. Ann Surg Oncol. 2012 Nov;19(12):3816-22.

32. T Z, R B-S, K F, Wm P (2001) Peripheral primitive neuroectodermal tumor: challenge for multimodal treatment. World J Surg 25(11):1367-72

33. Shamberger RC, LaQuaglia MP, Gebhardt MC et al. (2003) Ewing sarcoma/primitive neuroectodermal tumor of the chest wall: impact of initial versus delayed resection on tumor margins, survival, and use of radiation therapy. Ann Surg 238(4):563-7; discussion 567-568

34. Gaspar N, Hawkins DS, Dirksen U et al (2015) Ewing Sarcoma: current management and future approaches through collaboration. J Clin Oncol 33(27):3036-3046

35. Jürgens H, Etspüler G, Göbel U et al. (1988) Malignant peripheral neuroectodermal tumors. a retrospective analysis of 42 patients. Cancer 61(2):349-57

36. Ahmad R, Mayol BR, Davis M, Rougraff BT (1999) Extraskeletal Ewing's sarcoma. Cancer 85(3):725-731

37. Gururangan S, Marina NM, Luo X et al (1998) Treatment of children with peripheral primitive neuroectodermal tumor or extraosseous Ewing's tumor with Ewing's-directed therapy. J Pediatr Hematol Oncol 20(1):55-61

38. Castex M-P, Rubie H, Stevens MCG et al (2007) Extraosseous localized ewing tumors: improved outcome with anthracyclinesthe French society of pediatric oncology and international society of pediatric oncology. J Clin Oncol 25(10):1176-1182

39. Nesbit ME, Gehan EA, Burgert EO et al (1990) Multimodal therapy for the management of primary, nonmetastatic Ewing's sarcoma of bone: a long-term follow-up of the First Intergroup study. J Clin Oncol 8(10):1664-1674

40. Burgert EO, Nesbit ME, Garnsey LA et al (1990) Multimodal therapy for the management of nonpelvic, localized Ewing's sarcoma of bone: intergroup study IESS-II. J Clin Oncol 8(9):1514-1524

41. Grier HE, Krailo MD, Tarbell NJ et al (2003) Addition of ifosfamide and etoposide to standard chemotherapy for Ewing's sarcoma and primitive neuroectodermal tumor of bone. N Engl J Med 348(8):694-701

Publisher's Note Springer Nature remains neutral with regard to jurisdictional claims in published maps and institutional affiliations. 